\title{
OH/IR STARS AS TRACERS OF GALACTIC POPULATIONS
}

\author{
H.J.HABING \\ Sterrewacht Leiden
}

\begin{abstract}
I discuss observable quantities of $\mathrm{OH} / \mathrm{IR}$ stars and their interpretation. The strong $1612 \mathrm{MHz}$ maser is found in about $1 / 2$ of all the stars that reach the AGB and then have the following properties: they are oxygen-rich, of high-luminosity $\left(3,000 \rightarrow 30,000 L_{\odot}\right)$, vary in luminosity with a long period (P>500 d), lose mass rapidly $\left(\dot{M}>10^{-6} M_{\odot} /\right.$ yr $)$ and have a metallicity high enough to form sufficient amounts of dust (the metallicity of the LMC is probably the lowest value sufficient to produce OH/IR stars). OH/IR stars can be several Gyr old. There are at least three different populations each characterized by different galactic orbits. (1) OH/IR stars in the galactic disk on almost circular orbits; (2) OH/IR stars in the galactic bulge on orbits of lower angular momentum; (3) OH/IR stars within 150 pc from the galactic center forming a rapidly rotating system.
\end{abstract}

Key words: $\mathrm{OH} / \mathrm{IR}$ stars

\section{The transparancy of interstellar dust}

In Figure 1 I show an ESO photograph of an area of 60 degrees by 40 degrees around the center of our Galaxy- it contains most of the galactic bulge. The image is made up by stars and by interstellar matter and it is not nicely ellipsoidal. Clearly the picture is affected by the irregular distribution of the interstellar matter causing an intermingling of dark and bright areas. Drawn onto this figure are the many small regions where in recent times different observers have studied the stellar population of the bulge. The rectangle at the center represents the area covered by an infrared study by Catchpole et al. (1990; see Glass' review in these proceedings). Look at the distribution of these small regions: it shows the difficulty of penetrating, at visual wavelengths, the galactic center, that "heart of darkness". Studies of the stellar population in small windows as Sgr I and Baade's window are invaluable; yet there are too few windows to give us a convincing picture of how the properties of the stellar population vary over the bulge- interpolation between the various windows leaves too much uncertainty.

Dust absorption hides the inner Galaxy at all UV, visual and at the shortest infrared wavelengths; for wavelengths longer than a few microns the galactic plane is essentially transparent (also for hard X-radiation; much of what I will say is therefore also valid for X-ray sources). If we want to study the stellar population everywhere in the galactic bulge we have to study it where we can see the stars: in the infrared, at radio wavelengths, in $\mathrm{X}$ - rays. What populations of stars are there available?

\section{OH/IR stars : observable quantities, results, interpretation}

In this review I discuss stars that emit practically all their energy in the middleinfrared, and that have a remarkable, strong maser line of hydroxyl, $\mathrm{OH}$, at 1612 $\mathrm{MHz}$ or $18 \mathrm{~cm}$ : the $\mathrm{OH} / \mathrm{IR}$ stars. In addition the stars can be detected in a few other maser lines and in thermally excited molecular lines (especially $\mathrm{CO}$ and $\mathrm{SiO}$ ). 


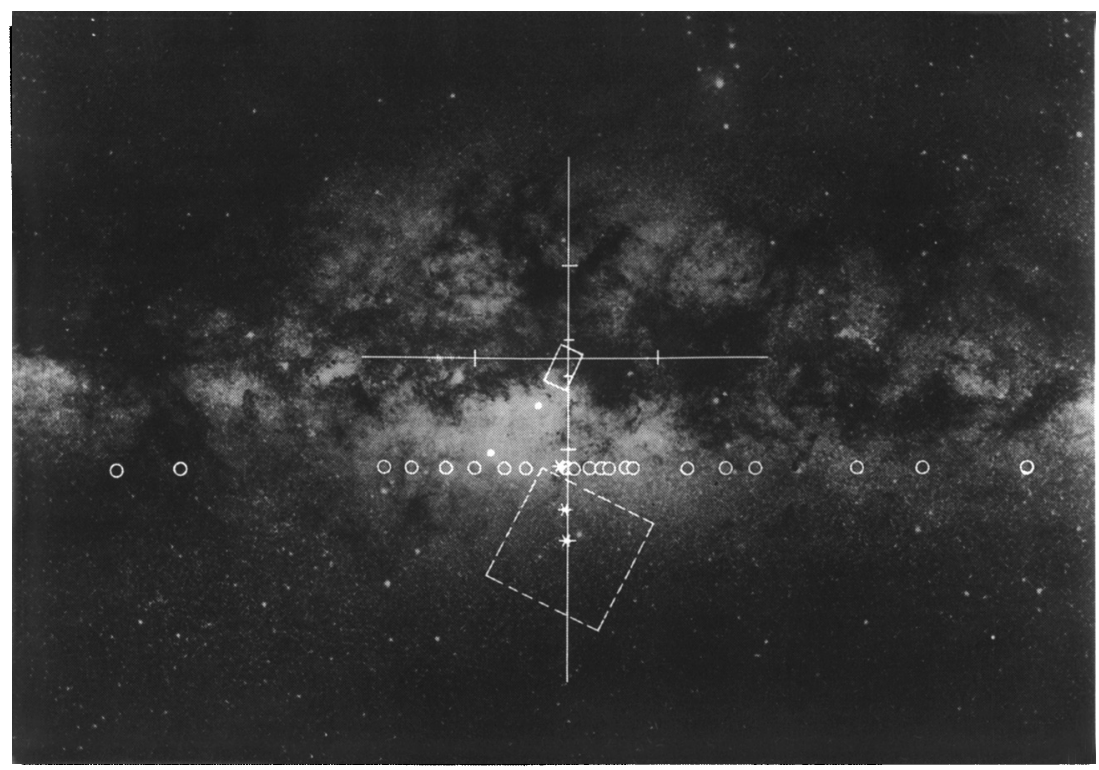

Fig. 1. An ESO photograph (The Messenger, 1986, 46, 14-15) of the galactic bulge. The axes show the galactic coordinate system $(1, b)$, the outermost tickmarks indicate $5^{\circ}$. The dashed square indicates the Palomar Groningen Field 3, and two of Baade's windows are indicated by filled circles. Terndrup's (1988) deep CCD photometry fields are indicated by asterisks; the open circles are Blanco and Terndrup's (1989) survey fields for late-type giants. The solid rectangle at the center is the field studied by Catchpole et al. (1990). (Courtesy: ESO and J. Blommaert)

I will discuss (1) stellar parameters that can be measured, (2) the nature of the stars (structure, luminosity) and (3) the question what galactic populations they represent (or equivalently: age and atomic composition). Readers who want a more elaborate introduction are referied to the proceedings of conferences dedicated to these and similar stars (e.g. Mennessier and Omont, 1990). In the next review Dejonghe discusses kinematical and dynamical conclusions about the bulge that are based on the observations of $\mathrm{OH} / \mathrm{IR}$ stars.

\subsection{Observable quantities}

In Figure 2a I show the (infrared) spectrum of one of the brightest $\mathrm{OH} / \mathrm{IR}$ stars, in figure $2 \mathrm{~b}$ its $1612 \mathrm{MHz}$ spectrum and in figure $2 \mathrm{c}$ the $\mathrm{CO}(2-1)$ emission line.

The infrared spectrum (figure $2 a$ ) is explained very well by spherically symmetric outflow of gas and dust around a late type giant: an elaborate modelling technique has been developped over the last 15 years (e.g. David and Papoular, 1992; Justtanont and Tielens, 1992). Figure 3 shows a collection of model fits to spectra for some M-type Miras and OH/IR stars. The models form a one dimensional series, with the optical depth in the silicate absorption band at $9.7 \mu \mathrm{m}$ increasing from the 
upper left to the lower right plot (Bedijn, 1987). From the observed spectrum one thus derives the value of the optical depth of the envelope, and $m_{\text {bol }}$, the apparent bolometric magnitude, by integration over all wavelengths (or $L_{*} / 4 \pi d^{2}$, where $L_{*}$ is the stellar luminosity and $d$ the distance).

The models just mentioned give a good representation of the spectra as observed. However, the calculations are based on an assumed density distribution and this distribution is not necessarily in agreement with the dynamical effects of radiation pressure: the photons transfer momentum to the dust particles and this momentum is transmitted by friction very efficiently to the gas. Recently Tignon, Tielens and I have made model calculations that simultaneously take into account the radiative transfer and the radiation pressure. Our specific aim was to study how the outflow velocity, $v_{\text {out }}$ depends on parameters such as the luminosity, $L_{*}$, the mass loss rate, $\dot{M}$, and the dust-to- gas ratio, $\delta$. Our main conclusion is that $v_{\text {out }} \propto L_{*}^{\frac{1}{3}} \delta^{\frac{1}{2}}$ and that $v_{\text {out }}$ is affected by $\dot{M}$ only when $\dot{M}<10^{-6} M_{\odot} /$ yr. This result agrees with the systematic properties measured for $v_{\text {out }}$ (see below). It then follows that an estimate of $\delta$ may be obtained once $v_{\text {out }}$ and $L_{*}$ have been measured. In oxygen-rich stars the dust consists of silicates and silicium is not created by fusion inside AGB stars, thus the value of $\delta$ is likely to represent the initial abundance of silicium. $\delta$ is a measure of the metallicity of the OH/IR star.

Here it is necessary to point out a persistent misunderstanding, or a plain error, that continues to appear in the litterature: The outflow of matter represents a flow of momentum equal to $\dot{M} v_{\text {out }}$; the radiation emitted by the star carries with it a momentum flow equal to $L_{*} / c$; let $\beta$ be the ratio between the two. Now it is often stated that $\beta \leq 1$ : the momentum flow of the photons has to exceed that of the gas "because the shell is radiation-driven". This conclusion is wrong: if the photons get reflected often enough $\beta$ might reach the value $\infty$ ! That in circumstellar shells $\beta$ may easily be larger than 1 has been argued in detail, and convincingly, by Gail and Sedlmayer (1986) and Lefèvre (1989), but statements to the contrary can be found in publications as recent as 1992 .

The maser line profile in figure $2 \mathrm{~b}$ is quite characteristic for $1612 \mathrm{MHz} \mathrm{OH} / \mathrm{IR}$ stars: two narrow peaks separated by many times the peak width; a sharp increase in flux at the outside of the peaks, but on the inner side a much more gentle slope. The line shape is quite well explained, qualitatively and quantitatively (Spaans and van Langevelde, 1992) by a spherical shell of hydroxyl molecules that are radiatively pumped by infrared emission from the center. Each of the two peaks defines a Doppler shifted velocity; the average of these two velocities is the stellar radial velocity, $v_{*}$; the difference equals twice the expansion velocity of the shell, $v_{\text {out }}$. The total line flux is another parameter that can be well measured. Theory and observations lead to the insight (see e.g. Elitzur, 1992) that the $1612 \mathrm{MHz}$ maser is pumped by $\mathrm{OH}$ line photons at 35 and at $53 \mu \mathrm{m}$, and it is predicted that the number of maser photons is a certain fraction of the number of pump photons. In recent years it has been shown that this is indeed the case; the efficiency of the conversion of pump into maser photons is a strong function of the (dust) optical 

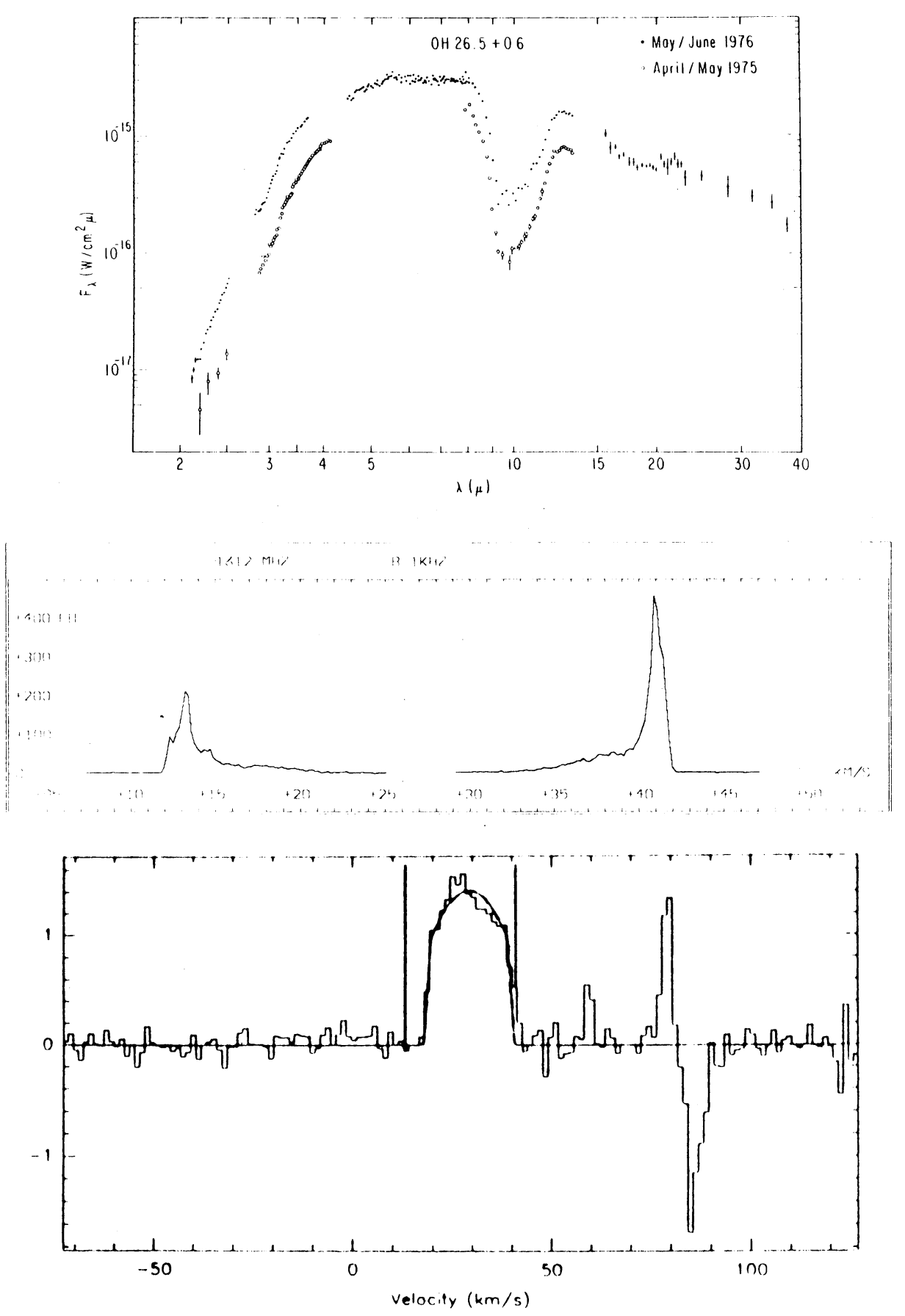

Fig. 2. The $\mathrm{OH} / \mathrm{IR}$ star $\mathrm{OH} 26.5+0.6$ or AFGL 2205. Figure 2a gives the infrared spectrum (Forrest et al., 1978); Figure 2b the $1612 \mathrm{MHz} \mathrm{OH}$ maser line (Andersson et al., 1974) and Figure $2 \mathrm{c}$ the $\mathrm{CO}(2 \rightarrow 1)$ line (Heske et al., 1990). 


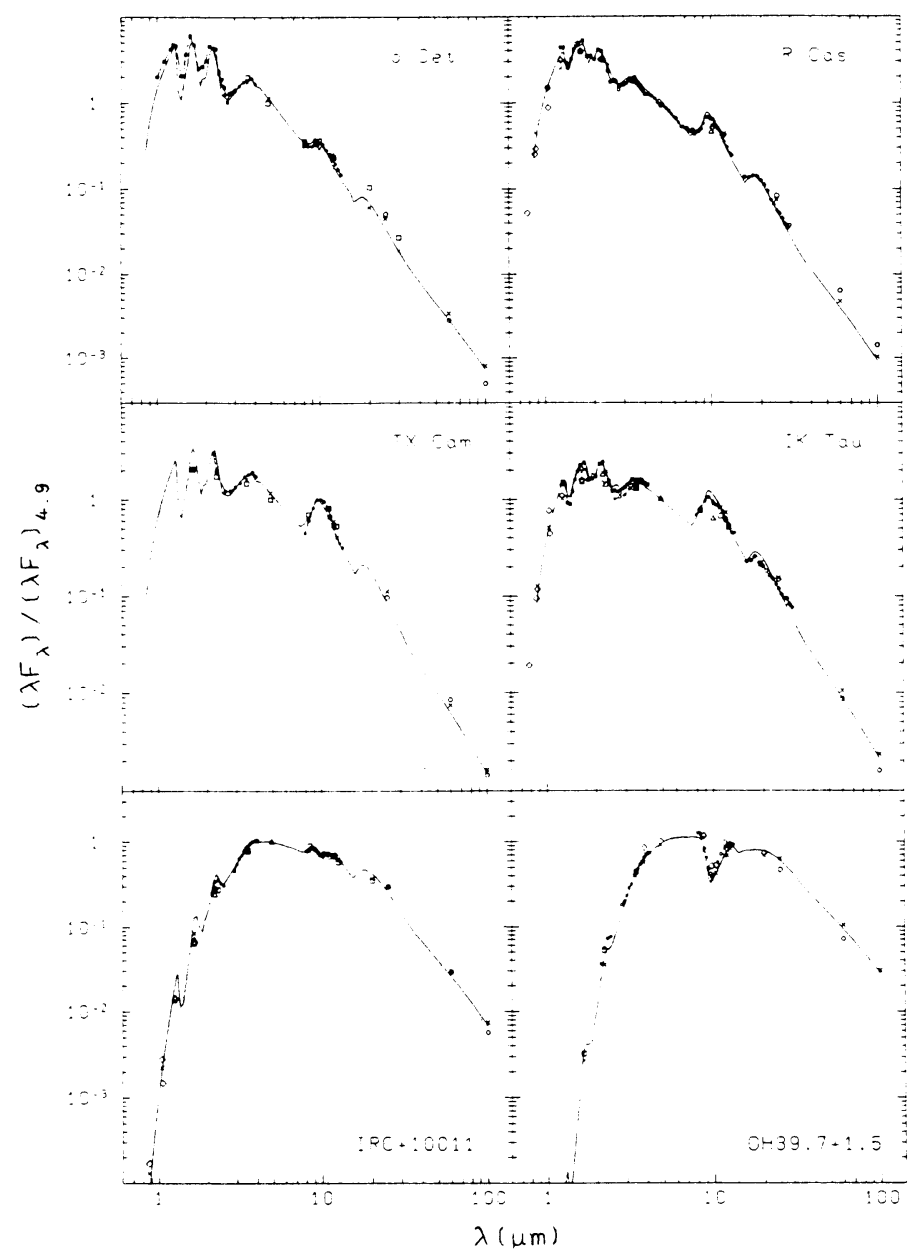

Fig. 3. Model fits to the spectra of M-type Miras and OH/IR variables. The models have optical depth in the silicate band ranging from 0.04 for the upper left to 10.0 for the lower right (Bedijn, 1987). Crosses denote calculated broad band photometric fluxes; other symbols observed photometric fluxes.

depth (Dickinson, 1991; Chengalur et al., 1992; Le Squeren et al., 1992) - and thus of the mass-loss rate. The ratio between the $\mathrm{OH} 1612 \mathrm{MHz}$ maser flux and the midinfrared flux is thus a measure of the mass-loss rate. Also the variation of the maser flux with time is interesting: this variation has a very long period and it is con sono with the infrared radiation. While it varies in intensity the line profile retains its shape, almost perfectly, but not completely: the blue shifted peak reaches maximum a few weeks (i.e. a few percent of the total period) before the red shifted peak. This phase difference between the light curves of the two peaks is explained by a light travel time effect and the value of the difference measures the linear diameter of the 


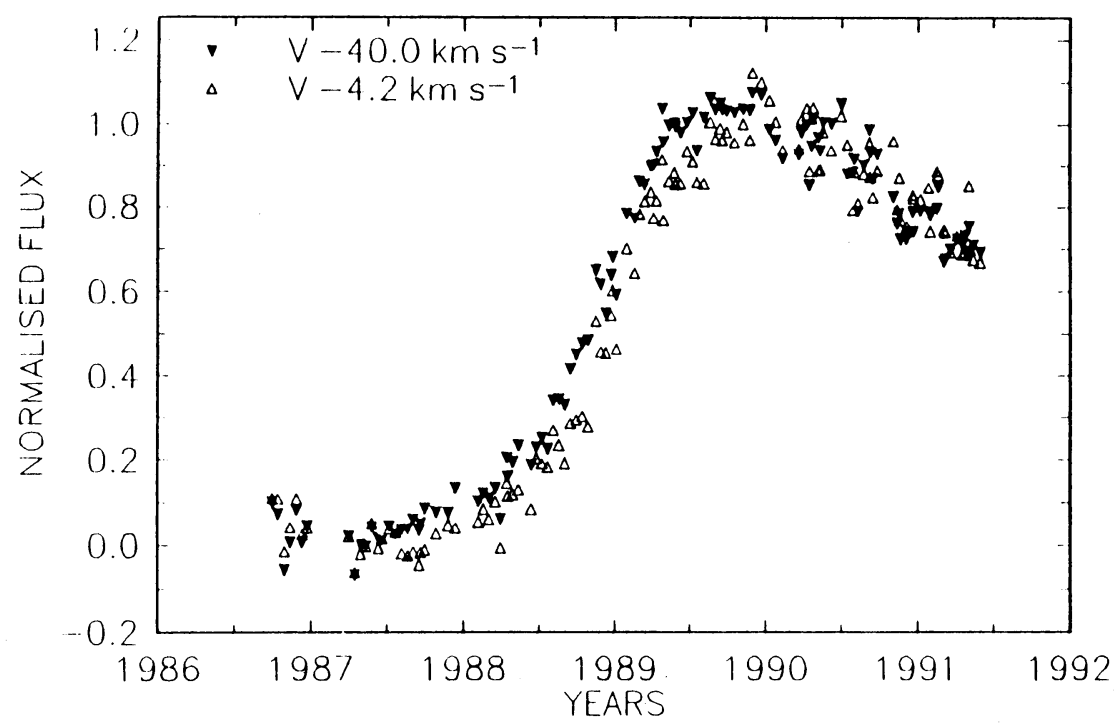

Fig. 4. Normalised light curves of blue-shifted $(\mathrm{V}=-40.0 \mathrm{~km} / \mathrm{s})$ and red-shifted $(\mathrm{V}=-4.2$ $\mathrm{km} / \mathrm{s}$ ) points in the 1612-MHz maser spectrum of OH357.3-1.3= RAFGL 5379 (West et al., 1992).

masering shell (typically a few lightweeks). By also measuring its angular diameter via interferometric techniques, one finds directly the stellar distance by a geometrical method. To apply the method much patience and a high accuracy is required; nevertheless distances have been measured to several OH/IR stars. In figure 4 one recognizes easily the phase differences between the red and blue lightcurves of a very strong, southern $\mathrm{OH} / \mathrm{IR}$ star at $1.4 \pm 0.1 \mathrm{kpc}$ (West et al., 1992). An attempt to measure the distance to OH/IR stars at the galactic center and thus to the center itself has failed, because unexpectedly strong inhomogeneities in the free electron distribution scatter the $18 \mathrm{~cm}$ radio waves and smear out the images of the OH/IR masers (van Langevelde et al., 1992). Nevertheless, one should continue to praise the very precise techniques of radio astronomy; there are still promises for the near future: Almost all $\mathrm{OH} / \mathrm{IR}$ stars produce also $\mathrm{SiO}$ masers at $\mathrm{mm}$ wavelengths; within a few years VLBI will become operational at $\mathrm{mm}$ wavelengths and then one can measure the $\mathrm{SiO}$ maser positions with an accuracy of a few tens of $\mu$ arcsec. This will allow the determination of proper motions of the masers even at the distance of the galactic center $(50 \mathrm{~km} / \mathrm{s}$ at $8,000 \mathrm{pc}$ corresponds to 1.25 marcsec /year $)$. Not only will such VLBI measurements give us three dimensional velocity information, but also will the statistical comparison of radial velocities with the proper motions give us a new, independent distance determination to this group of stars, that is: to the galactic center.

Figure $2 c$ shows an observed emission line from thermally excited CO-molecules. 
Several other lines of this molecule have been detected too; the same is true for several other molecules (e.g. $\mathrm{SiO}$ ). The lines of $\mathrm{CO}$ are formed at large distances from the star $\left(10^{17} \mathrm{~cm}\right)$ whereas the $\mathrm{SiO}$ lines are formed much deeper inward (between $10^{14}$ and $10^{16} \mathrm{~cm}$ ). The parabolic shape of the line profile is typical for dense circumstellar, expanding envelopes. From the line profile one measures the stellar velocity, the expansion velocity and, from the integrated line flux and only if the stellar distance is known, the total number of molecules in the envelope. Model calculations are essential for the interpretation. Recently the models for the CO-lines have been greatly improved (at the cost of increased complexity) by calculating the excitation temperature of the molecules in a self consistent manner (Sahai, 1990; Kastner, 1992). Large systematic samples of observations of circumstellar CO have been published by Margulis et al. (1990) and by Nyman et al. (1992).

Finally: spectrographs in the ISO satellite, to be launched in late 1995, may detect various absorption bands (e.g. vibrational $\mathrm{SiO}$ lines) that originate in the stellar atmosphere and not in the envelope; this will give a first glimpse of the star in the center of the circumstellar envelopes.

\subsection{RESUlTS FROM MEASUREMENTS}

\subsubsection{Source lists}

$\mathrm{OH} / \mathrm{IR}$ stars are strong masers; the first $500 \mathrm{OH} / \mathrm{IR}$ stars have been detected by searching in an unbiased way in the $1612 \mathrm{MHz}$ line; for a compilation of these early detections see te Lintel Hekkert et al. (1989). This search technique was slow. But OH/IR stars are also strong mid-infrared sources: they are powered by giant stars and all radiative energy is emitted in the mid-infrared. A succesful search technique is to select IRAS point sources and to observe these in the $1612 \mathrm{MHz}$ line. Over 1000 stars have thus been found through surveys with the Parkes and Effelsberg telescopes (te Lintel Hekkert et al., 1991); with the Arecibo telescope (Eder et al. 1988, Lewis et al., 1990, Chengular et al., 1993) and with the Nançay telescope (Le Squeren et al., 1992). In all these surveys the detection probablity never rose about $1 / 2$; why it does not equal 1 is an unexplained fact at the moment (see e.g. Gaylard et al., 1989, and Lewis, 1992). IRAS was confused in the galactic plane, especially in the inner Galaxy, and there one expects the $\mathrm{OH} / \mathrm{IR}$ source density to be highest. Thus the one thousand stars detected in the IRAS directed surveys form an essentially incomplete sample; even worse, the sample is incomplete by an unknown factor. Recently two new unbiased and ambitious $1612 \mathrm{MHz}$ line surveys have been started in the galactic plane using the VLA to the north and the AT to the south of the galactic center. In total there are now over $1500 \mathrm{OH} / \mathrm{IR}$ stars known.

\subsubsection{Mass loss rates, $\dot{M}$}

From the observed outflow velocities and from the dust and gas density in the circumstellar envelope one obtains an estimate of the mass outflow rate- see e.g. some recent determinations by Schutte and Tielens (1989), Sahai (1990), Justtannont and Tielens (1992), and Kastner (1992). Values are found between $10^{-7}$ and 
$10^{-4} M_{\odot} / y r$. In a small but significant fraction of the stars the circumstellar shell is detached and at some distance from the star- suggesting strongly that the mass loss process has stopped abruptly less than $10^{5} \mathrm{yr}$ ago. The first examples of detached shells were recognized in carbon-rich stars (Willems and de Jong, 1988; Olofsson et al., 1990), but recently it has been shown that also oxygen-rich stars show detached shells (Zijlstra et al. 1992).

\subsubsection{Outflow velocity, $v_{\text {out }}$}

Analysis of the $1612 \mathrm{MHz}$ line profile in some $800 \mathrm{OH} / \mathrm{IR}$ stars shows a range of velocities between 10 and $20 \mathrm{~km} / \mathrm{s}$ (te Lintel Hekkert, 1990). CO- line observations give the same values; a few, possibly interesting discrepancies have been reported. The range of outflow values is thus small, and yet it appears to be significant whether the velocity is at the low end of the range or at the high end. Evidence derived from OH/IR stars in the Magellanic Clouds (Wood et al., 1992), in the galactic anticenter (Blommaert et al., 1993), close to the galactic center (Lindqvist et al., 1992), all indicate that $v_{\text {out }}$ is higher when the luminosity is higher and/or when the metallicity is higher. These systematic trends can be understood if the circumstellar shell is accelerated by radiation pressure (see the discussion in section 2.1). In less reddened, related objects with $1612 \mathrm{MHz}$ maser emission (Mira variables) the outflow velocities range between a few and $10 \mathrm{~km} / \mathrm{s}$ and are well correlated with the stellar period (Sivagnanam et al. 1989). The correlation disappears for periods over 500 days, that is: for OH/IR stars.

\subsubsection{Luminosities, $L_{*}$}

Luminosities are derived from the observed flux $\left(L_{*} / 4 \pi d^{2}\right)$, after measuring the distance. In principle the best (geometrical) distance determination is by the phaselag method: see above. A statistical approach was taken by Habing (1988), who studied the distribution of the OH/IR stars in the Galaxy; the only scale length that enters the analysis is the distance from the Sun to the galactic center. A third way to overcome the distance problem is by using the OH/IR stars in the galactic bulge - they are all at the same distance within $25 \%$. The net result is that luminosities have been found betweeen 3,000 and $30,000 L_{\odot}$, with an average between 5,500 and 8,000 $L_{\odot}$ (van der Veen and Habing, 1990; Whitelock et al. 1991). A recent analysis of the new complete set of OH/IR stars discovered in Arecibo (Chengular et al., 1993) confirms these results.

\subsubsection{Variability}

For a significant number of $\mathrm{OH} / \mathrm{IR}$ stars flux density variations have been monitored (and periods have been determined) in the $\mathrm{OH}$ - line or at some IR wavelengthradio and infrared fluxes vary in phase (Harvey et al., 1974). The shortest periods are around 500 days; the longest now known is over 3,000 days (Herman and Habing, 1985; West et al., 1992). In the galactic bulge the longest period is around 1000 days (Whitelock et al., 1991); longer periods are found only in stars belonging to the galactic disk. Herman and Habing (1985) studied the variation in a sample of 35 
$\mathrm{OH} / \mathrm{IR}$ stars in the disk of the Galaxy; about $1 / 4$ showed small or no variation at all. These non-variables proved to have IRAS colours much redder and quite different from those of the variable OH/IR stars (Olnon et al., 1984); this led Bedijn to the suggestion that the non-variable stars had stopped - quite abruptly - to lose matter (Habing et al. 1987). The best known example of these non-variable stars is $\mathrm{OH} 17.7-2.0$, now generally considered to be an object in transition from the AGB to the a planetary nebula phase. This however is a phase of very short duration and if the ratio of one non-variable out of every four OH/IR stars will stand further scrutiny, the total $\mathrm{OH} / \mathrm{IR}$ phase can last only a very short time. Recent results by van Langevelde (1992) from monitoring OH/IR stars at the galactic center also show up a significant fraction of non- variables.

\subsubsection{Related Objects}

In the IRAS atlas of low resolution spectra (IRAS-LRS) there are over 2000 objects in the classes 2, 3 and 6, and a large fraction of these are stars with circumstellar envelopes containing silicate dust (in a recent paper Omont et al., 1993, have argued that LRS spectra 21 and 22 correspond to very cool carbon-rich stars rather than to oxygen-rich stars). Not all objects have been identified and studied in detail; yet it seems safe to assume that also in other properties they are related; Mira variables are at one end of the sequence, where the circumstellar envelope is thin and the $\mathrm{OH} / \mathrm{IR}$ stars are at the other end, where the envelope has become very thick. Mira variables thus appear to be related to the OH/IR stars. It is tempting to speculate that Miras ultimately develop into $\mathrm{OH} / \mathrm{IR}$ stars and that all $\mathrm{OH} / \mathrm{IR}$ stars have been Miras in the beginning, but this is not at all certain.

Another group of relatives are the carbon stars: from the carbon-mira's to dust enshrouded stars like IRC+10216. The relation between "oxygen-rich" stars (such as Mira's and $\mathrm{OH} / \mathrm{IR}$ stars) and these carbon-rich stars is a question that has evoked much discussion over the last few years; in this (ongoing) discussion the common ground is the prediction by the theory of stellar evolution that an oxygenrich star can turn into a carbon-rich star when during a thermal pulse freshlyproduced carbon is injected into the hydrogen-rich envelope and when this injection upsets the C/O-ratio.

\subsection{INTERPRETATION}

What are $\mathrm{OH} / \mathrm{IR}$ stars? A standard answer begins by noting that $\mathrm{OH} / \mathrm{IR}$ stars share important properties with the Mira variables, and that the differences are quantitative and not qualitative. Miras have late M-spectra; they are long period variables (periods between 200 and 500 days) with large amplitudes (1 to 2 bolometric magnitudes); they lose significant amounts of mass $\left(10^{-7}\right.$ to $\left.10^{-8} M_{\odot} / \mathrm{yr}\right)$ at a low outflow velocity (3 to $10 \mathrm{~km} / \mathrm{s})$; masers $\left(\mathrm{OH}, \mathrm{H}_{2} \mathrm{O}\right.$ and $\left.\mathrm{SiO}\right)$ are formed at some distance from the photospheric surface; luminosities are between 3000 and $10,000 L_{\odot} . \mathrm{OH} / \mathrm{IR}$ stars share all these properties but have more extreme values (luminosities from 3,000 to $30,000 L_{\odot}$; periods from 500 to 3,000 days; mass loss rate from $10^{-6}$ to $10^{-4} M_{\odot} / \mathrm{yr}$; outflow velocity from 10 to $20 \mathrm{~km} / \mathrm{s}$ ). We miss one 
essential piece of information about the OH/IR stars: the photospheric temperature; we cannot observe the star directly, only its circumstellar envelope. In view of the similarities with Miras one assumes that a star of very late spectral type is also present at the center of an $\mathrm{OH} / \mathrm{IR}$ star.

Late-type stars of such high luminosities must be AGB stars or supergiants. Model calculations (e.g. Iben, 1991) indicate that AGB stars consist of a core of significant mass, but of very small radius and consisting of carbon, oxygen and a degenerate electron gas. This core is surrounded by a huge envelope of hydrogen gas. Hydrogen is burned into helium in a very thin layer surrounding the core; when the mass of helium becomes larger than a critical value the helium burns into carbon in a very short time ("thermal pulse"). The core mass thus grows all the time and because the luminosity is proportional to the core mass, the luminosity increases as well; this growth is exponential with a time scale that is the same for stars of all masses: $\tau_{\text {nucl }} \approx 10^{6} \mathrm{yr}$. The evolution of the star is determined by this growing core (well studied in hydrostatic models) and by mass loss on the outside. Mass loss is a hydrodynamic process that unfortunately can not (yet?) be calculated from first principles, but that we know to exist from observations. Mass-loss has a time scale $\tau_{\mathrm{ml}}=M / \dot{M} ;$ if $\dot{M}$ exceeds values like $10^{-6} M_{\odot} /$ yr then for a star of only a few solar masses one might have $\tau_{\text {nucl }}<\tau_{\text {ml }}$. In that case the star will lose its outer envelope faster than its core can grow and consequently it bleeds to death while its luminosity stays constant. This is the evolutionary stage to be associated with $\mathrm{OH} / \mathrm{IR}$ stars and their relatives. It is clear that the precise chain of events in these last phases of the stellar existence depends critically on how the mass loss process evolves in time and of this history we are not yet certain. A new trend is to assume that mass loss is an interrupted process- the suggestion comes from the observations of detached shells (see above). It is tempting to associate such an interruption with the occurrence of a thermal pulse. This suggestion leads to a further speculation: At what luminosity the first thermal pulse will occur is determined by the main sequence mass of what is now the OH/IR star. Suppose that this thermal pulse inititiates the phase of rapid mass loss; then the luminosity of the star is arrested and the luminosity of an $\mathrm{OH} / \mathrm{IR}$ star thus equals its luminosity at the moment of its first thermal pulse; we can thus estimate the main-sequence mass from the luminosity. Ideas similar to this have been worked out quantitatively by Vassiliadis and Wood (1993) who combine calculations of the stellar interior with an empirical (and still somewhat uncertain) mass loss rate.

In the circumstellar envelope radiation pressure is bound to be a dominating dynamic process once the dust grains have been formed; however it is clear that radiation pressure is not the cause of the loss of mass: Condensation of the dust particles takes place at large distances from the star and if the star had a hydrostatic atmosphere the density of gas at the condensation point would be much too low to lead to a significant rate of loss of mass. The pulsating stellar atmosphere is, however, strongly dynamic and not at all static and Bowen and Willson (1991) argue rather convincingly that stellar pulsations and the effects these have on the atmosphere lead to a sufficient enlargement of the atmospheric scale height (the same conclusions had been reached earlier by Bedijn, 1988).

In summary, what stars do become OH/IR stars? In addition to the arguments just 
presented there are more clues: (1) in the anticenter only young and massive stars are $\mathrm{OH} / \mathrm{IR}$ stars whereas (2) in the galactic bulge relatively low luminosity objects show the strong $\mathrm{OH} 1612 \mathrm{MHz}$ maser, and these objects have galactic orbits that can in no way be associated with Population I. Thus I suggest that all those stars become $\mathrm{OH} / \mathrm{IR}$ star that reach the AGB, that pulsate sufficiently strongly, and that have a metallicity high enough to produce the required amount of dust. In the next section I will argue that OH/IR stars are found not only among population I objects, but also among older populations.

\section{OH/IR stars as tracers of galactic populations}

In the next review Dejonghe will discuss the kinematic properties of the total sample of OH/IR stars. As an introduction a few remarks: The concept of "stellar populations" is difficult to use. Yet it appears to me that in the set of 1500 known $\mathrm{OH} / \mathrm{IR}$ stars we can distinguish three groups, each with its own spatial distribution and its own kinematic signature, and hence each can be called "a population":

1. Stars belonging to the galactic disk moving on almost circular orbits. Except for the very near ones they are all at small latitudes. There are very few outside the solar circle, that is at $|l|>90^{\circ}$. The radial velocities indicate large angular momenta, that is, they follow galactic rotation closely, although an "older" subgroup (smaller angular momentum, less concentrated to the galactic plane) and a "younger" subgroup can be recognised using the value of $v_{\text {out }}$ as criterion (Baud et al., 1981; Chengalur et al., 1992). The first (1612 MHz) surveys for OH/IR stars in the late seventies revealed especially these disk stars and therefore the (now inadequate) notion rose that all OH/IR stars belong to Population I. Disk $\mathrm{OH}$ stars can be very luminous: probably up to the AGB luminosity limit of about $40,000 L_{\odot}$ and beyond that as supergiants. Some AGB members have very long periods, between 1000 and 3000 days. I tentatively estimate that about $2 / 3$ to $3 / 4$ of the 1500 known belong to the disk.

2. Stars in inner parts of the galaxy on orbits of low angular momentum, possibly outlining the bulge.

Tentatively their number is estimated at about $1 / 4$ to $1 / 3$ of the total number of 1500 . There is no sharp criterion to distinguish the bulge stars on an individual basis from the disk stars- the distinction is made on statistical grounds (te Lintel Hekkert et al., 1991). The analysis of these stars (determination of luminosity and of pulsational period) is incomplete; the paper by van der Veen and Habing (1990) is only a first step. The stars studied by Whitelock et al. (1991; see also her review in these proceedings) are closely related to $\mathrm{OH} / \mathrm{IR}$ stars, but have lower mass loss rates. One conclusion can be drawn: the total luminosity of an individual bulge star does not exceed $10,000 L_{\odot}$ and its pulsational period is below 1000 days; more luminous $\mathrm{OH} / \mathrm{IR}$ stars are found only in the disk population.

3. Stars very close to the galactic center, that is within $150 \mathrm{pc}$.

There are some 140 known (Lindqvist et al., 1992). Kinematically they are very different from the bulge as they rotate rapidly around the center 
(see the next review by Dejonghe). Infrared observations, essential to the determination of their luminosities, have been hindered by serious confusion problems and became succcesful only recently (Blommaert, 1992). The luminosities are modest (below 10,000 $L_{\odot}$ ), and the pulsational periods below 1000 days (van Langevelde et al., 1993). A surprising property is shown in figure 5. A relatively large fraction of the galactic centre population consists of stars with $\mathrm{v}_{\text {out }}>20 \mathrm{~km} / \mathrm{s}$, in the range of supergiants. Yet, the luminosities show that they are not supergiants and we take this as an indicator that the dust-to-gas ratio, $\delta$, is higher in this rapidly rotating galactic population. One then speculates that this holds also for the silicium abundance (and for the overall metallicity?). On the basis of the facts just presented Blommaert (1992) speculates that these OH/IR stars might be $10^{10}$ yr old. An additional interesting set are the three stars discovered and discussed by van Langevelde et al. (1992) that have a radial velocity differing by more than $3 \sigma$ from the mean: true high-vlocity stars. To explain this high velocity the authors suggest that these stars are on very elongated, almost radial orbits, seen near their pericenter where the velocity is highest. Because OH/IR stars represent a large population of less evolved stars, actually a fraction significantly larger than $3 / 140$ of the central population might be on such radial orbits.

After having discussed the places in the Galaxy where one finds the OH/IR stars it is illustrative to discuss the places where one does not find them. Surprisingly few $\mathrm{OH} / \mathrm{IR}$ stars have been found outside the solar circle. The ones known appear to be very young and luminous and to have low outflow velocities: indicative of a young age and yet a low metallicity. This, and the fact that in the direction of the outer Galaxy carbon stars are as abundantly present as in the direction of the galactic center, suggests that low metallicity (1) forces a star of medium to low mass to become a carbon star before it can become an OH/IR star and/or (2) does not support an $\mathrm{OH}$ maser. Finally: what about the halo? I do not know of any OH/IR star that might be a halo object; the detection of IRAS source SSC $08546+1732$, a cool envelope around a carbon-rich star in what is probably a halo object (Cutri et al. 1989) is most intriguing.

\section{Conclusions}

$\mathrm{OH} / \mathrm{IR}$ stars are oxygen-rich AGB stars with heavy mass loss and with a sufficient large oxygen and silicium abundance (= exceeding the solar value?). More than 1500 of them are known, and many more remain to be detected. The total sample can be divided into three groups each with its own location in the Galaxy and with its own kinematic behaviour; each group its own population:

- $\quad \mathrm{OH} / \mathrm{IR}$ stars belonging to the galactic disk

- $\quad \mathrm{OH} / \mathrm{IR}$ stars belonging to the bulge

- $\mathrm{OH} / \mathrm{IR}$ stars belonging to the nucleus of the Galaxy.

The bulge stars show little rotation, but the stars of the nucleus show fast rotation. In addition, the stars of the nucleus are probably supermetal rich. 


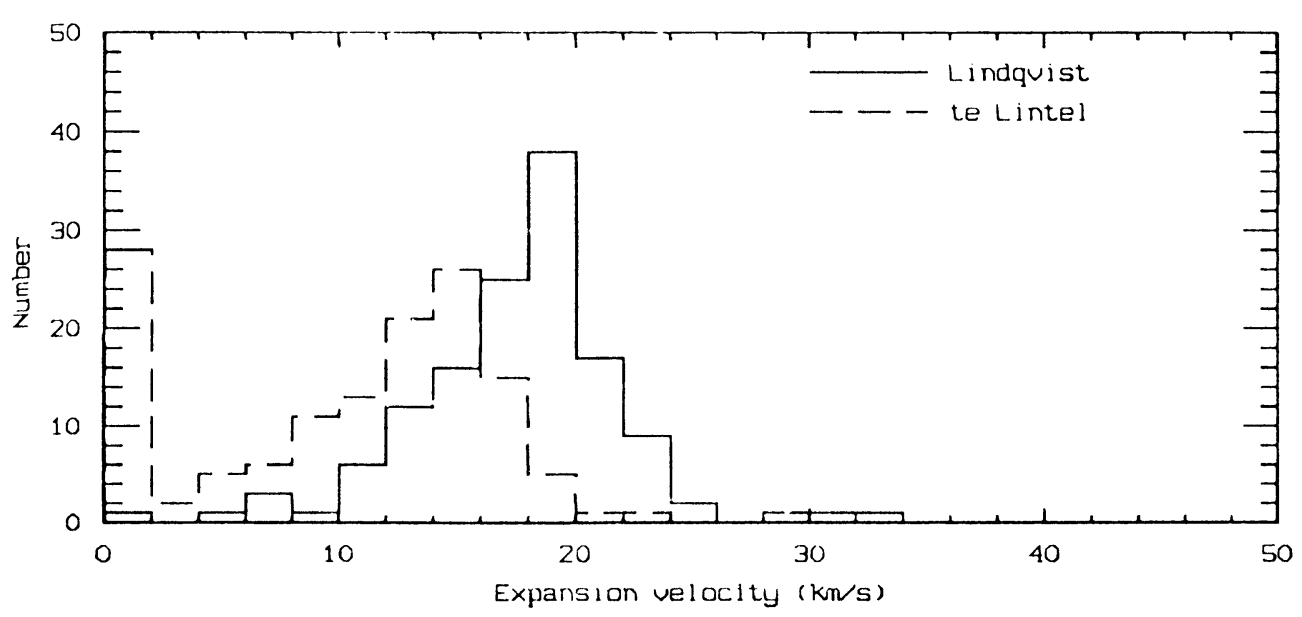

Fig. 5. A histogram of the expansion velocity for two different samples of both $134 \mathrm{OH} / \mathrm{IR}$ stars; the Lindqvist et al. (1992) sample within $1^{\circ}$ of the centre and part of the te Lintel et al. (1991) sample within $4^{\circ}$.

\section{References}

Andersson, C., Johansson,L.E.B., Goss, W.M., Winnberg, A., N-Q Rieu, 1974, Astron. Astrophys. 30, 475.

Baud, B., Habing, H.J., Matthewson, H.E., Winnberg, A. 1981, Astron. Astrophys. 95, 156.

Becker, R.H., White, R.L., Proctor, D.D. 1992, Astron. J. 102, 538.

Bedijn, P.J. 1987 Astron. Astrophys. 186, 136.

Bedijn, P.J. 1988 Astron. Astrophys. 205, 105.

Blommaert, J.A.D.L. 1992, thesis, Leiden University.

Blommaert, J.A.D.L., van der Veen, W.E.C.J., Habing, H.J. 1993, Astron. Astrophys. 267, 39.

Bowen, G.H., Willson, L.A. 1991, Astrophys. J. Lett. 375, L53.

Catchpole, R.M., Whitelock, P.A., Glass, I.S. 1990, Mon. Not. Roy. Astr. Soc. 247, 479.

Chengalur, J.N., Lewis, B.M., Eder, J., Terzian, Y. 1992, preprint.

Cutri, R.M., Low, F.J., Kleinmann, S.G., Olszewski, E.W., Willner, S.P., Campbell, B., Gillett, F.C. 1989, Astron. J. 97, 866.

David, P., Papoular, R. 1992, Astron. Astrophys. 256, 183. Dickinson, D.F. 1991, Astrophys. J. Lett.379, L29.

Eder, J., Lewis, B.M., Terzian, Y. 1988, Ap.J. Suppl. 66, 183.

Elitzur, M. 1992, "Astronomical Masers" (Kluwer Academic Publishers).

Forrest, W.J., Gillett, F.C., Houck, J.R., McCarthy, J.F., Merrill, K.M., Pipher, J.L., Puetter, R.C., Russell, R.W., Soifer, B.T., Willner, S.P. 1978, Ap. J. $219,114$.

Gail, H.-P., Sedlmayr, E. 1986 Astron. Astrophys. 256, 201.

Gaylard, M.J., West, M.E., Whitelock, P.A., Cohen, R.J. 1989, Monthly Not. Roy. Astr. Soc. 236, 247.

Habing, H.J. 1988, Astron. Astrophys. 200, 40.

Habing, H.J., van der Veen, W.E.C.J., Geballe, T. 1987, in "Late Stages of Stellar Evolution", eds. S. Kwok and S.R. Pottasch, Reidel, Dordrecht, p. 91. 
Harvey, P.M., Bechis, K.B., Wilson, W.J., Ball, J.A. 1974, Astrophys. J. Suppl. 27, 331.

Herman, J., Habing, H.J. 1985, Astron. Astrophys. Suppl. 59, 523.

Heske, A., Forveille, T., Omont, A., Sivagnanam, P., van der Veen, W.E.C.J., Habing, H.J., 1990 Astron. Astrophys. 239, 173.

Iben, I. 1991, Astrophys. J. Suppl. Ser. 76, 55.

Justtanont, K., Tielens, A.G.G.M. 1992, Astrophys. J. 389 , 400.

Kastner, J.H. 1992, Ap. J. $401,337$.

Lefèvre, J., 1989 Astron. Astrophys. 219, 265.

Le Squeren, A.M., Sivagnanam, P., Dennefeld, M., David, P. 1992, Astron. Astrophys. 254, 133.

Lewis, B.M. 1992, Astrophys. J. 396, 251.

Lewis, B.M., Eder, J., Terzian, Y. 1990, Astrophys. J. 362, 634.

Lindqvist, M., Habing, H.J., Winnberg, A. 1992, Astron. Astrophys. 259, 118.

Margulis, M., Van Blerkom, D.J., Snell, R.L., Kleinmann, S.G. 1990, Astrophys. J. 361, 663.

Mennesier, M.O., Omont, A. (editors) 1990, "From Mira's to Planetary Nebulae: Which path for stellar evolution?" Editions Frontières (Gif-sur-Yvette).

Nyman, L.-Å., Booth, R.S., Carlsström, U., Habing, H.J., Heske, A., Sahai, R., Stark, R., van der Veen, W.E.C.J., Winnberg, A. 1992, Astron. Astrophys. Suppl. Ser. 92, 43.

Olofsson, H., Carlström, U., Eriksson, K., Gustafsson, B., Willson, L.A. 1990, Astron. Astrophys. 230, L13.

Omont, A., Loup, C., Forveille, T., te Lintel Hekkert, P., Habing, H., Sivagnanam, P. 1993, Astron. Astrophys. 267, 515.

Olnon, F.M., Baud, B., Habing, H.J., de Jong, T., Harris, S., Pottasch S.R. 1984, Astrophys. J. Lett. 278, L41

Sahai, R. 1990, Astrophys. J. $362,652$.

Schutte, W.A., Tielens, A.G.G.M. 1989, Astrophys. J. 343, 369.

Sivagnanam, P., Le Squeren, A.M., Foy, F., Tran Minh, F. 1989, Astron. Astrophys. 211, 341.

Spaans, M., van Langevelde, H.J. 1992, Mon. Not. Roy. Astr. Soc. 258 , 159.

te Lintel Hekkert, P., Caswell, J.L., Habing, H.J., Haynes, R.F., Norris, R.P. 1991, Astron. Astrophys. Suppl. Ser. 90, 327.

te Lintel Hekkert, P., Dejonghe, H., Habing, H.J. 1991, Proc. Astron. Soc. Australia, 9, 20.

van Langevelde, H.J. 1992, thesis, Leiden University.

van Langevelde, H.J., Frail, D.A., Cordes, J.M., Diamond, P.J. 1992, Astrophys. J. 396, 686.

van Langeveldè, H.J., Janssens, A.M., Goss, W.M., Habing, H.J. Winnberg, A. 1993, Astron. Astrophys. Suppl. (in press).

Vassiliadis, E., Wood, P.R. 1993, Astrophys. J. (in press).

van der Veen, W.E.C.J., Habing, H.J. 1990, Astron. Astrophys. 231, 404.

West, M.E., Gaylard, M.J., Combrinck, W.L., Cohen, R.J., Sheperd, M.C. 1992, in "Variable Stars and Galaxies", ed. B. Warner (Astronomical Society of the Pacific Conference Series no 30) page 277.

Whitelock, P.A., Feast, M.F., Catchpole, R. 1991, Mon. Not. Roy. Astr. Soc. 248, 276.

Willems, F.J., de Jong, T. 1986, Astrophys. J. Lett. 309, L39.

Wood, P.R., Whiteoak, J.B., Hughes, S.M.G., Bessell, M.S., Gardner, F.F., Hyland, A.R. 1992, Astrophys. J. (Letters) 397, 552.

Zijlstra, A.A., Loup, C., Waters, L.B.F.M., de Jong, T. Astron. Astrophys. 265, L5. 


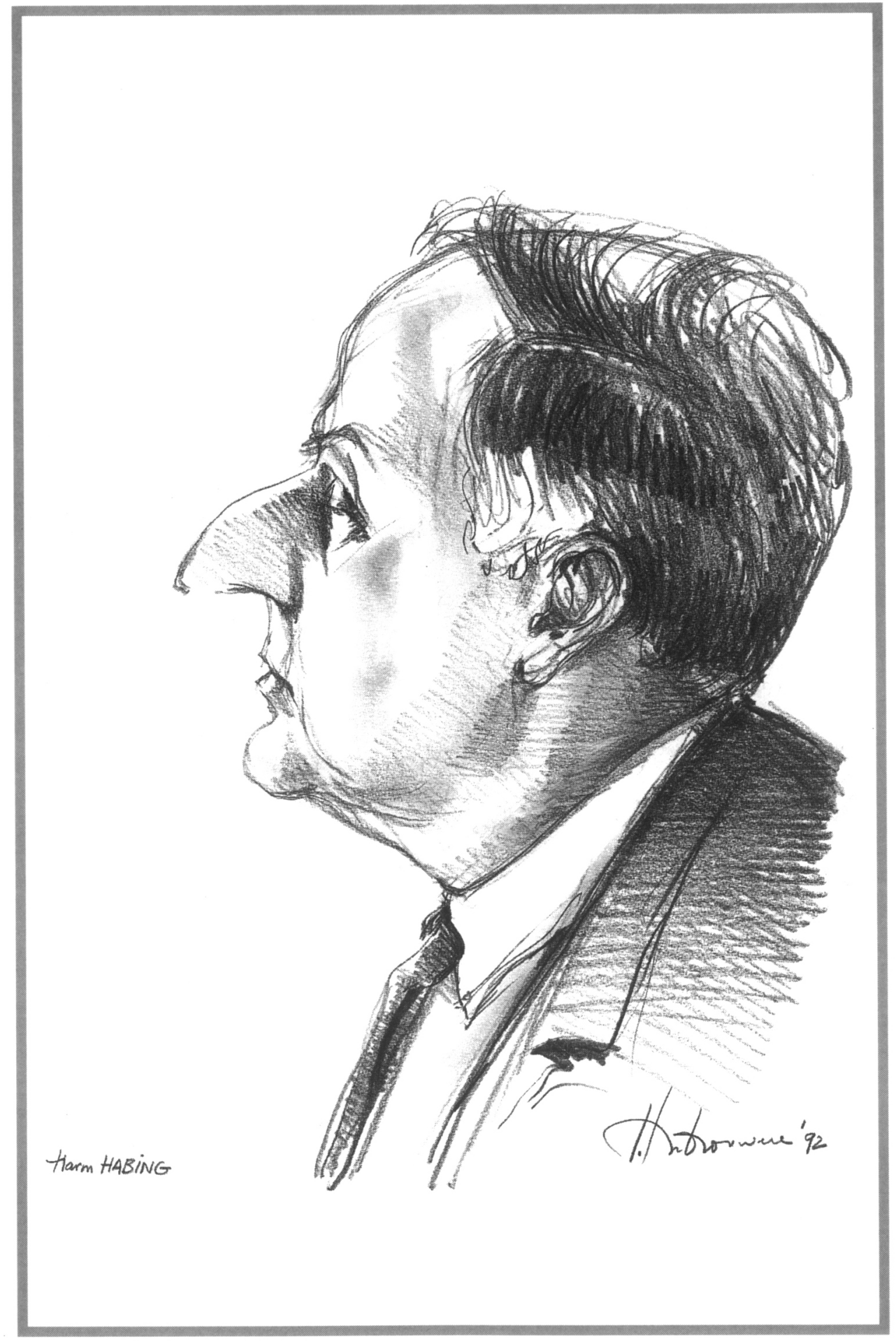




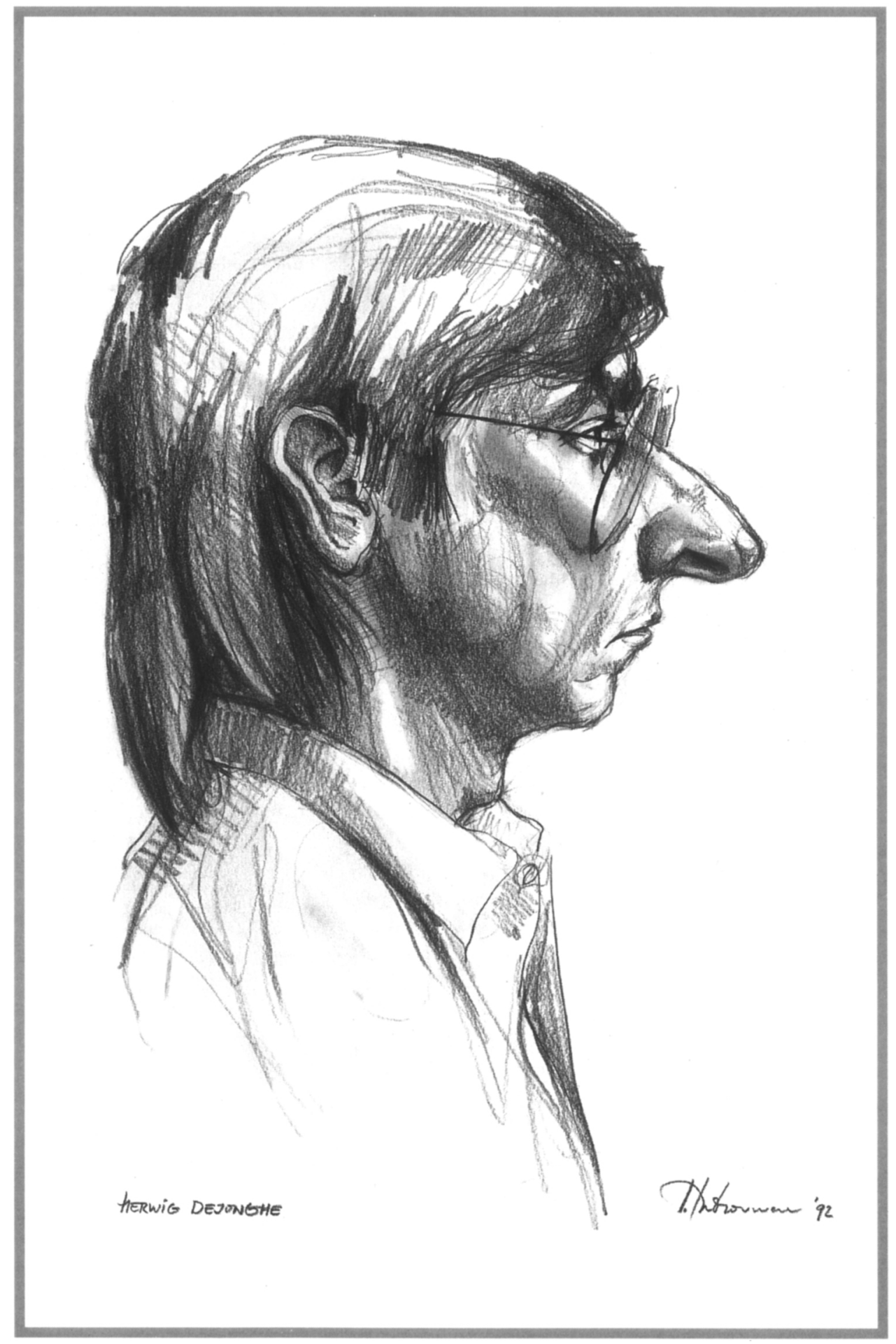

